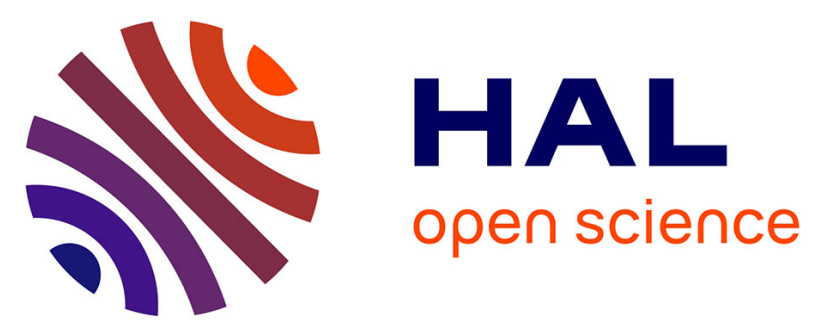

\title{
3D Elastic FWI with a Non-Linear Model Constraint: Application to a Real Complex Onshore Dataset
}

Phuong-Thu Trinh, Romain Brossier, Laurent Lemaistre, Ludovic Métivier, Jean Virieux

\section{- To cite this version:}

Phuong-Thu Trinh, Romain Brossier, Laurent Lemaistre, Ludovic Métivier, Jean Virieux. 3D Elastic FWI with a Non-Linear Model Constraint: Application to a Real Complex Onshore Dataset. 81st EAGE Conference and Exhibition 2019, Jun 2019, London, United Kingdom. 10.3997/22144609.201901344. hal-02325662

\section{HAL Id: hal-02325662 https://hal.science/hal-02325662}

Submitted on 24 Nov 2020

HAL is a multi-disciplinary open access archive for the deposit and dissemination of scientific research documents, whether they are published or not. The documents may come from teaching and research institutions in France or abroad, or from public or private research centers.
L'archive ouverte pluridisciplinaire HAL, est destinée au dépôt et à la diffusion de documents scientifiques de niveau recherche, publiés ou non, émanant des établissements d'enseignement et de recherche français ou étrangers, des laboratoires publics ou privés. 


\title{
3D elastic FWI with a non-linear model constraint: Application to a real complex onshore dataset
}

\author{
Phuong-Thu TRINH ${ }^{1,2}$, Romain Brossier ${ }^{2}$, Laurent Lemaistre ${ }^{1}$, Ludovic Métivier $^{2,3}$, and Jean Virieux ${ }^{2}$ \\ 1 Total E\&P, F-64000 Pau, France \\ ${ }^{2}$ Univ. Grenoble Alpes, ISTerre, F-38058 Grenoble, France \\ ${ }^{3}$ Univ. Grenoble Alpes - CNRS, LJK, F-38058 Grenoble, France
}

January 14, 2019

Question to response in the abstract submission:

Main objectives Multi-parameter full waveform inversion in onshore targets remains very challenging. By using a spectral-element-based approach for accurate elastic wave simulation, the paper focuses on the challenges of the elastic multi-parameters reconstruction in mountain areas. Through a real foothill data application, the paper highlights the importance of a mixture of strategies in both data and model domains for a successful inversion.

New aspects covered The paper considers the simultaneous reconstruction of $\mathrm{P}$ - and $\mathrm{S}$-wave components in a 3D elastic full waveform inversion for a real foothill target. The paper belongs to one of very first FWI works in complex onshore areas. Apart from the data complexity and elastic multi-parameter FWI problem, we have to deal with various practical issues such as a significant amount of noise in the data, sparse acquisition and topography representation uncertainty. We illustrate how additional prior information such as well logs and geological knowledge can be used to assist the FWI workflow. We also present a non-linear model constraint on the ratio $\mathrm{Vp} / \mathrm{Vs}$ to suppress unrealistic model updates, introduced by the ill-posed inversion process. Our progressive time-windowing data injection helps to mitigate the cycle-skipping issue at the later arrivals and maximize the considering data quantity, and eventually improve the model estimation.

\begin{abstract}
(193 words - limit is 200 words)

Full waveform inversion (FWI) of 3D onshore targets is very challenging due to the complex freesurface-related and heterogeneities effects. In such areas, the seismic wavefield displays converted and back-scattering energy, with strong contribution of surface waves. A time-domain spectral-elementbased approach is used for accurate elastic wavefield simulation in mountain areas. The challenges of the elastic multi-parameter FWI in complex land areas are highlighted through a real foothill dataset. Various practical issues such as a significant amount of noise in the data, sparse acquisition and topography representation uncertainty must be considered. We illustrate how additional prior information such as well logs and geological knowledge can be used to assist the FWI workflow. First, a non-linear model constraint on the ratio $\mathrm{Vp} / \mathrm{Vs}$, relying on the logs information, may better constrain the Vs based on the stable Vp estimation. Second, appropriate gradient preconditioning design is also crucial for meaningful model parameters reconstruction. At the current stage, the inversion mainly focuses on the early-body waves, which are gradually incorporated through a progressive time-windowing. The strategy helps to mitigate the cycle-skipping issue at the later arrivals and maximize the considering data quantity, and eventually improve the model estimation.
\end{abstract}




\section{Introduction}

Full waveform inversion (FWI) for onshore targets is very challenging due to complex free-surface-related effects and 3D geometry representation. In such areas, the seismic wavefield is dominated by highly energetic and dispersive surface waves, converted waves and back-scattering energy when the waves hit the steep slopes at the surface, or strong velocity contrasts. These complex effects cannot be fully removed or compensated by data pre-processing, implying that a correct description of the physics is strongly advisable for accurate model parameters estimation. Moreover, considering the complete physical phenomena of the wave propagation would make possible to take benefit of each piece of recorded data, for expected more accurate results and higher resolution. Adequate geometry representation is also required to honor the interaction between elastic waves and geological structures such as rapid topography variation and erosions. When considering complex structures or geological heterogeneities, regularization and preconditioning strategies also play an important role to mitigate the ill-posedness of the inversion problem (Guitton et al., 2012; Trinh et al., 2017).

Our previous studies have demonstrated the possibility to recover simultaneously $V_{p}$ and $V_{s}$ from various synthetic benchmarks (Trinh et al., 2018, 2019). In this study, we investigate a 3D multi-parameter elastic FWI workflow on a 2D real seismic line, acquired from a complex foothill area with significant topography variation. The sub-surface is characterized by complex faulting, significant folding and highly dipping structures. The rough topography variation and the excessive amount of noises make the standard velocity model building approach very difficult, for instance velocity picking and tomography techniques. The work is performed using our SEM46 code (Spectral Element Method for Seismic Imaging in eXploration), based on a time-domain spectral element method (SEM) (Trinh et al., 2019).

\section{Wave propagation and FWI in SEM46}

The code uses a flexible Cartesian-based deformed mesh with high-order geometry representation to capture complex topographies and variable element-size to reduce the numerical cost as illustrated in Figure 1 (Trinh et al., 2019). The wavefield at the boundary is absorbed by a combination of sponge layers and radiative boundary condition (Trinh, 2018). The inversion is based on the minimization of the least-squares norm between the observed and the calculated data. The FWI gradient preconditioning is directly performed on the SEM mesh, by using the structurally-based nonstationary and anisotropic Bessel smoothing filter (Trinh et al., 2017). The filter shape is defined by variable coherence lengths: $L_{v}(\mathbf{x})$ is associated with the direction perpendicular to the local bedding plan, $L_{u}(\mathbf{x})$ and $L_{w}(\mathbf{x})$ are related to the planar structure of potential geological features. The $3 \mathrm{D}$ orientation is controlled by azimuth and dip angles.

Non-linear model constraint on the ratio $V_{p} / V_{s}$

Elastic FWI applications with the least-squares misfit function is generally more sensitive to $V_{s}$. When considering significant geological heterogeneities, the inversion process

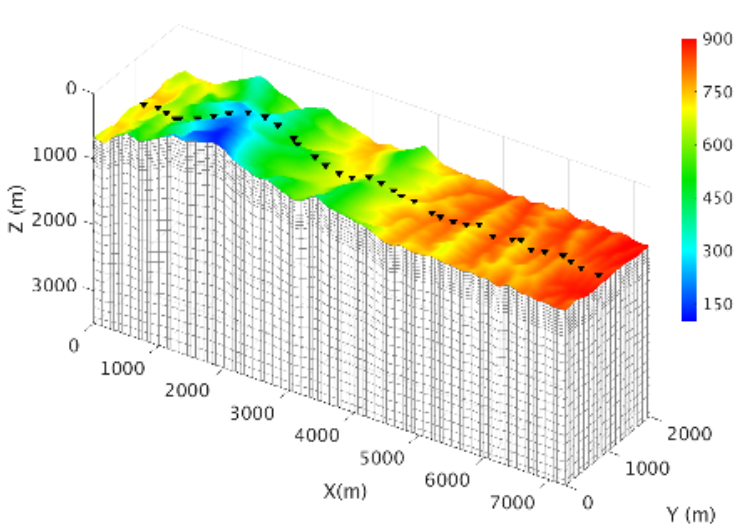

Figure 1 The 3D SEM mesh designed at $4 \mathrm{~Hz}$ maximal frequency: 62.5 m elements size at the near surface and 175 m elements size at the deeper part. The topography map is put on top of the mesh, where the colorbar illustrates the absolute depth $Z_{a b s}(m)$ from a pre-defined datum. The source positions of the $2 D$ line are indicated by black triangles $(\Delta S=200 \mathrm{~m})$. might lead to unrealistic updates where $V_{s}>V_{p}$ or too small value of $V_{s}$. Therefore, additional prior information should be incorporated in addition to the standard gradient smoothing strategy to further guide the inversion process.

We introduce a non-linear constraint on the ratio $V_{p} / V_{s}$, which is applied simultaneously with the bound constraints on the range of $V_{p}$ and $V_{s}$. The misfit function is now minimized over a restricted model space $\left(\mathscr{C}_{1} \cap \mathscr{C}_{2}\right)$, which is the intersection of two convex sets:

- Bound constraint $\mathscr{C}_{1}: V_{p}$ and $V_{s}$ should vary within pre-defined ranges,

- Ratio constraint $\mathscr{C}_{2}$ : The ratio $V_{p} / V_{s}$ should vary within a pre-defined range.

These constraints can be designed based on well logs or simple geological knowledge about the structure. The projection of model parameters onto the intersection of two constraints are performed through the Dykstra's algorithm, which ensures the uniqueness of the solution, independently with the order of the constraints application (Peters and Herrmann, 2017; Trinh, 2018). 
Figure 2 Gradient preconditioning design: A - The dip field: The first $1 \mathrm{~km}$ below the free-surface is extracted from the initial $V_{p}$; $B$ - The cartoon to illustrate the effect of the smoothing operator onto the gradient.
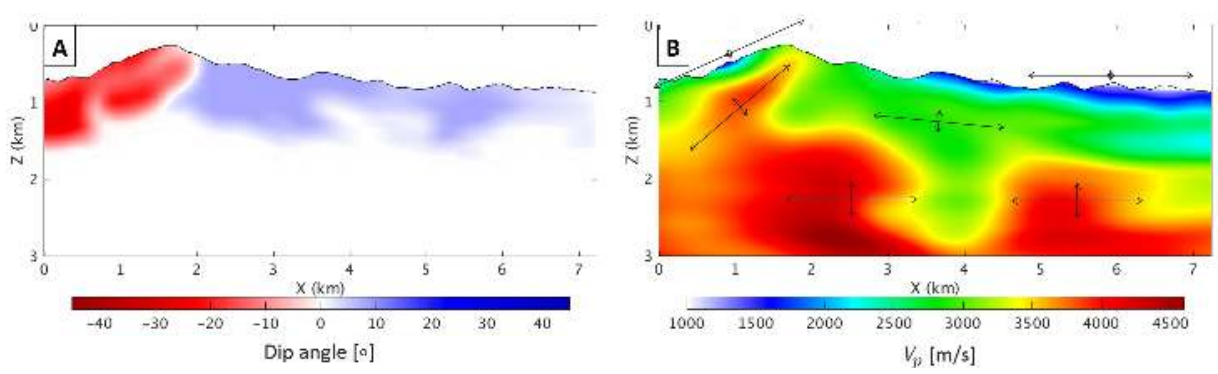

\section{Foothill dataset configuration}

The considered 2D line is located across the mountain trend with significant topography change as shown in Figure 1. We limit the investigated area at $7.2 \mathrm{~km}$ in the $x$-direction. The entire seismic line is acquired with explosive sources, buried at $7 \mathrm{~m}$ depth. This source type produces un-controlled source signature but it also generates the low frequency content, down to $2 \mathrm{~Hz}$, which is beneficial for FWI. All receivers are single vertical $(z)$ component, located at the free-surface. The sources and receivers follow the same line, as indicated by the black triangles in Figure 1. The acquisition has significant deviation in the $y$ direction, at about $400 \mathrm{~m}$, especially when crossing the mountain. This deviation implies that the 3D effect consideration is advisable for any imaging technique. The source and receiver spacings are not regular with rather sparse distribution: the average distance between adjacent receivers is approximately $37 \mathrm{~m}$, and about $40 \mathrm{~m}$ between adjacent sources.

\section{Inversion setup}

Since our SEM46 tool can perform true-amplitude and waveform elastic waves propagation, we do not apply any sophisticated processing sequences such as static correction or surface waves removal. We only remove the low frequency noises below $2 \mathrm{~Hz}$ by a high-pass filter. The data within the range [2-4 Hz] is considered for inversion, which provides a reasonable signal to noise ratio.

\section{Initial models \& Mesh design}

The initial $V_{p}$ model is provided by the data owner as shown in Figure 4A. The initial $V_{s}$ and density models are computed from the given $V_{p}$ through some empirical relationships, obtained from upscaling logs data. The initial models quality control shows acceptable starting data fit for the selected frequency range. To account for the 3D propagation effect, we consider a narrow 3D model with $2 \mathrm{~km}$ in $y$-direction. Due to the limitation of $7.2 \mathrm{~km}$ offset in $x$-direction, we do not expect any model update below $3.5 \mathrm{~km}$ in depth. Since we expect significant heterogeneities at the near-surface, the first $500 \mathrm{~m}$ in $z$-direction is filled with small elements. The element sizes are computed from the expected shear wavelength. The mesh design for the maximal frequency at $4 \mathrm{~Hz}$ is shown in Figure 1. Each element is deformed by $4^{\text {th }}$-order shape functions to describe the sharp topography (Trinh et al., 2019).

\section{Gradient preconditioning and model constraints}

To prepare for any rapid vertical velocity variation at the near-surface, the preconditioning has highly anisotropic shape at this area with vertical coherence length being $L_{v}=16 \mathrm{~m}$, and horizontal coherence lengths as $L_{u}=200 \mathrm{~m}$. It should be noted that $L_{v}$ is equivalent to a quarter of the element size at the near-surface (62.5 $\mathrm{m}$ as shown in Figure 1) and a large value of $L_{u}$ is used to attenuate the acquisition footprint due the sparse source spacing. In the deeper part with bigger element size, we use larger coherence lengths with $L_{v}=70 \mathrm{~m}$ and $L_{u}=150 \mathrm{~m}$.

The highly anisotropic smoothing at the near-surface requires an "appropriate" rotation design, otherwise the preconditioning operator might introduce thin pinch-out artifacts following unexpected direction. The initial models provides rather good velocity trends, correctly following the topography and the expected

Figure 3 The design of model constraint on the ratio $V_{p} / V_{s}$ based on wells data. A tight constraint on the ratio $V_{p} / V_{s}$ is considered at the reservoir level normally associated with large $V_{p}$ value, whereas a less strict constraint is applied at the near-surface.

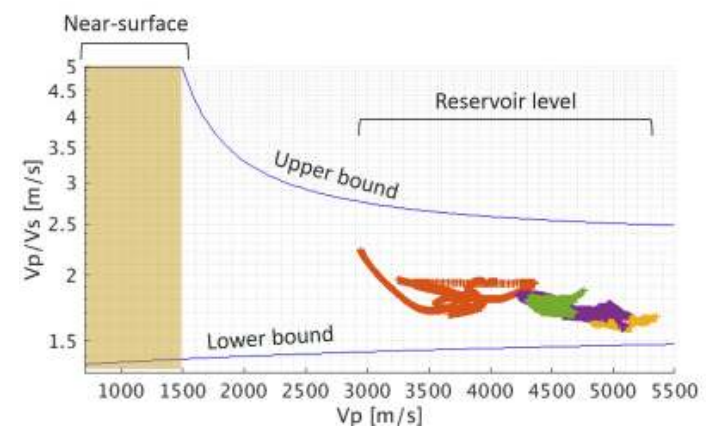


structures orientation for the first $1 \mathrm{~km}$. We thus compute the near-surface dip field from the initial $V_{p}$ and the deeper part is set to $0^{\circ}$ as shown in Figure 2A. The filtering orientation is illustrated in Figure 2B. When inverting simultaneously for $V_{p}$ and $V_{s}$, we suffer from the instability of the $V_{s}$ reconstruction meanwhile the $V_{p}$ remains stable. This comes from the lack of shear information in the explosive seismic data. At each spatial position $\mathbf{x}$, we thus constraint the estimated $V_{s}(\mathbf{x})$ through the inverted $V_{p}(\mathbf{x})$ following the non-stationary constraint on the ratio $V_{p} / V_{s}(\mathbf{x})$. A tight constraint on this ratio is considered at the reservoir level, normally associated with large $V_{p}$ value, whereas a less strict constraint is applied at the near-surface as shown in Figure 3. The upper and lower bounds are computed from $V_{p}$ (Trinh, 2018).

\section{Inversion workflow}

At this stage, we only consider the early body waves packages, arriving before the surface waves. The challenging incorporation of surface waves is under investigation. A simple bottom mute is used to separate the early body waves and surface waves. In the body waves packages, the cycle-skipping issue occurs for the later arrivals but not for the early arrivals. We thus design a progressive time-window to incorporate gradually the later arrivals into the inversion process. Each time-window is defined by a pair of top and bottom mutes, designed from the first-break, given by the data owner. The top mute is important to remove the coherent noises arriving before the first arrival. The bottom mute increases progressively by a half of the apparent wavelength per time-window.

The inversion contains different sequences, each being performed over one time-window with 4 iterations of the preconditioned $l$-BFGS optimization method. Within each sequence, the density and the source wavelet are kept unchanged. Since a relative short data interval with few traces is considered as the FWI input, we keep relatively small number of iterations per sequence. After each inversion sequence, the source wavelet is re-estimated, and the density is computed from the obtained $V_{p}$ model.

\section{Inversion results \& Discussion}

The inversion process makes some significant amplitude changes in both $V_{p}$ and $V_{s}$ models as shown in Figure 4B. The main fault trend which already existed in the initial model is preserved in the inverted models. The trend is further refined in the inverted $V_{p}$, and the high $V_{p}$ value is propagated up to the free-surface, which is coherent with the geological observation. The inversion also remove all the low- $V_{p}$ values at the near-surface, and considerably increases the $V_{p}$ background.

The inverted $V_{s}$ is more difficult to interpreted since it has a considerable amount of artifacts. This is due to the fact that the early body waves, acquired by the explosive sources, bring limited shear information. Even if the main fault direction is presented, the dimming of the estimated $V_{s}$ at the top of the mountain, at $X \approx 1.8 \mathrm{~km}$, is not coherent with the high velocity trend in $V_{p}$. The inverted $V_{s}$ detects some interesting low velocity trends at the near-surface, indicated by the black arrows in Figure 4. Their coherent appearance, size and amplitude in various FWI setting make us believe that they are geologically correct. The geological interpretation of the area indeed shows some possible near-surface ruptures. Since the available logs data are not located along the seismic line, they cannot be used to control the quality of inverted models.

An about $100 \mathrm{~m}$ thickness low-velocity layers underneath the free-surface appears in the $V_{s}$ model, as indicated by the red arrow in Figure 4B. This low-velocity layer does not have coherent shape and has never been detected by the $V_{p}$ estimation through our various FWI settings. Its appearance is not supported by any geological evidence. This inconsistency makes us believe that it is not geological correct.

Figure 5 shows the comparison of the maximal $4 \mathrm{~Hz}$ real data with the calculated computed from initial

Figure 4 Left - $V_{p}$ target crosssection; Right - $V_{s}$ target crosssection. A - Initial models; $B$ - Inverted models. Possible near-surface ruptures are indicated by the black arrows. Low-velocity $V_{s}$ layer at the free-surface with about $100 \mathrm{~m}$ thickness is indicated by the red arrow.
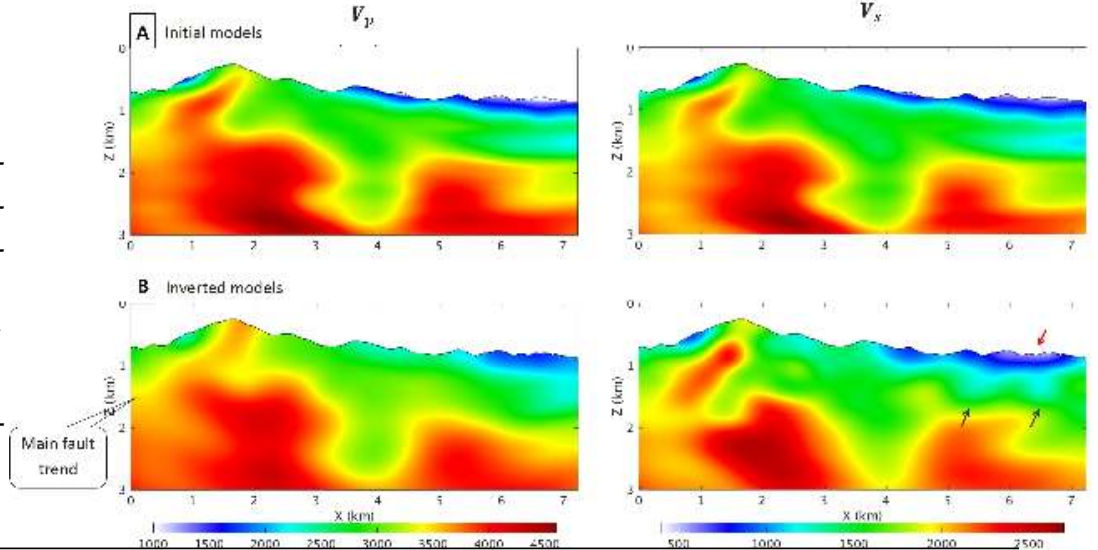


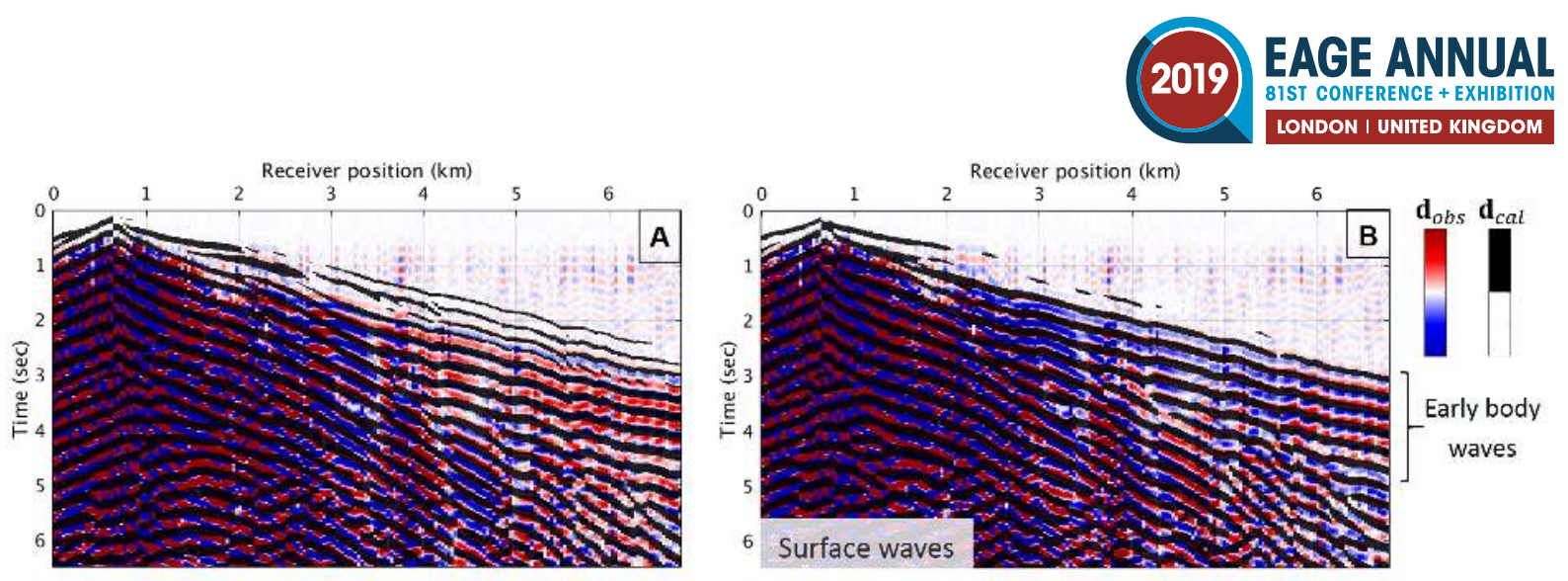

Figure 5 Data comparison for the first shot between the observed data $\mathbf{d}_{\text {obs }}$ and calculated data $\mathbf{d}_{\text {cal }}$ : (A) $\mathbf{d}_{\text {cal }}$ estimated from initial models in Figure $4 A,(B) \mathbf{d}_{\text {cal }}$ estimated from inverted models in Figure $4 B$. Observed data is displayed with red-and-blue color. Calculated data is displayed with black and transparent color. Good data-fit is identified when the red loops are perfectly covered by the black loops.

models and inverted models in Figure 4. Compared with the real data, the initial models produce relatively good wavefield prediction, with coherent body and surface waves. However, the early body waves package is cycle-skipped, apart from some first arrivals (Figure 5A). By injecting real data progressively through the progressive time-widows strategy, we improve the early body waves fitting as indicated by Figure 5B. We seem to also have a better surface waves prediction and mode conversion, for example at around $3 \mathrm{~km}$ position. However, the predicted data is almost cycle-skipped at the far offsets, at $6 \mathrm{~km}$ position. This issue is also detected in other shot gathers which further confirms the $100 \mathrm{~m}$ near-surface anomaly in $V_{s}$ in Figure 4B. We have investigated these near-surface artifacts by a synthetic feasibility study. It seems that such artifacts are caused by the acquisition mis-positioning in depth, coming from the surface elevation measurement error: The source depth available in the data SEGY header (GPS information) and the LIDAR topography maps are not totally consistent.

\section{Conclusions and perspectives}

The presented application illustrates the challenges in a real foothill environments. Apart from the data complexity and elastic multi-parameter FWI problem, we have to deal with various practical issues such as a significant amount of noise in the data, sparse acquisition and topography representation uncertainty. We illustrate how additional prior information such as well logs and geological knowledge can be used to assist the FWI workflow. A $V_{p}$-dependent non-linear model constraint on the ratio $V_{p} / V_{s}$, relying on the $\operatorname{logs}$ information, is considered to constrain the $V_{s}$ based on the stable $V_{p}$ estimation. In the next steps, we plan to consider more shots to compensate for the data lacking, also to investigate on alternative misfits design to better handle the cycle-skipping issue. However, a carefully re-processed data at might help to improve the inverted models quality.

\section{Acknowledgements}

This study was partially funded by TOTAL and the SEISCOPE consortium (http://seiscope2.osug.fr), sponsored by AKERBP, CGG, CHEVRON, EQUINOR, EXXON-MOBIL, JGI, PETROBRAS, SCHLUMBERGER, SHELL, SINOPEC and TOTAL. This study was granted access to the HPC resources of CIMENT infrastructure (https://ciment.ujf-grenoble.fr) and CINES/IDRIS/TGCC under the allocation 046091 made by GENCI. We thank TOTAL for providing the HPC resources and permission to present this work.

\section{References}

Guitton, A., Ayeni, G. and Díaz, E. [2012] Constrained full-waveform inversion by model reparameterization. Geophysics, 77(2), R117-R127.

Peters, B. and Herrmann, F.J. [2017] Constraints versus penalties for edge-preserving full-waveform inversion. The Leading Edge, 36(1), 94-100.

Trinh, P.T. [2018] 3D Multi-parameters Full Waveform Inversion for challenging land targets. Ph.D. thesis, University Grenoble Alpes.

Trinh, P.T., Brossier, R., Métivier, L., Tavard, L. and Virieux, J. [2019] Efficient 3D time-domain elastic and viscoelastic Full Waveform Inversion using a spectral-element method on flexible Cartesian-based mesh. Geophysics, 84(1), R75-R97.

Trinh, P.T., Brossier, R., Métivier, L. and Virieux, J. [2018] Data-oriented strategy and Vp/Vs model-constraint for simultaneous Vp and Vs reconstruction in 3D viscoelastic FWI: Application to the SEAM II Foothills dataset. In: 88th SEG Conference and Exhibition 2018, Anaheim.

Trinh, P.T., Brossier, R., Métivier, L., Virieux, J. and Wellington, P. [2017] Bessel smoothing filter for spectral element mesh. Geophysical Journal International, 209(3), 1489-1512. 